\title{
A New External Force for Snake Algorithm Based on Energy Diffusion
}

\author{
Mahdi A. Nisirat
}

\begin{abstract}
Object shape detection and localization techniques that utilize snake deformable models are one of the most promising image detection techniques. The binary edge maps, derived from the original image, are basically the class acted upon by the snake to extract the desired features. As a result, high and low energy content pixels are obtained. The high energy pixels are the pixels that reflect the object borders of a given image. This paper addresses a new external force that is calculated from the energy diffusion of high content pixels and is used to balance the internal forces of the snake. The proposed scheme showed better results in terms of computation speed and capture range than standard snake models. On the basis of the concavity convergence, analogous results are achieved in the proposed scheme compared with standard models.
\end{abstract}

Index Terms-Algorithms, computer vision, deformable models, feature extraction, image edge detection.

\section{INTRODUCTION}

Snakes are parametric curves that are initialized in the image plane and are allowed to evolve under the influence of internal and external forces to converge towards specific features in the image. The internal forces are computed from the snake to control its elasticity and consistency whereas the external forces are computed from the image. Extra constraint forces might be added to drive the image to converge to specific shapes in higher level image processing. The next subsection will present the different types of snakes that were developed and their advantages.

\section{Snake Algorithm}

Kass et al. [1], proposed that snakes are a class of active contours that consist of a set of points represented parametrically by their position vector. These points are allowed to move in the image domain by functional energy minimization so that they converge to the region of interest.

The functional energy to be minimized is basically formed from two parts: internal and external components. The internal energy component $E_{i n t}$ is calculated by iterative processes such as the Kass method, whereas the external component $E_{e x t}$ is computed using the desired features of the image. $E_{\text {int }}$ governs the shape and elasticity of the snake, whereas $E_{e x t}$ is used to attract the snake to the desired borders. Moreover, the internal energy component will give rise to bending forces that control the bending

Manuscript received on September 19, 2018; revised April 16, 2019

M. A. Nisirat is with the Faculty of Engineering Technology, Albalqa Applied University, Amman, Jordan (e-mail: mamnisirat@bau.edu.jo, mamnisirat@gmail.com). ability of the snake, and elasticity forces that hold the snake together during its movement. The external energy component will give rise to attractive forces that drive the snake to the desired feature. Additionally, an extra term of the energy minimization equation is the constraint energy $E_{c o n}$ term. This term should be imposed either by the user or by higher level processes to control the convergence of the snake to the feature. The functional energy relation is expressed as

$$
E=\int_{0}^{1}\left\{E_{\text {int }}(v(s))+E_{\text {ext }}(v(s))+E_{\text {con }}(v(s)) d s\right\}
$$

where $v(s)$ is the parametric representation of the snake $[x(s), y(s)]$, and $s \in[0,1]]$.

Since the early introduction of snake algorithms, researchers have proposed different external forces to attract the snake to the desired features in a given image. For example, Cohen and Cohen [2], [3] proposed a potential force to attract the snake into the suggested features. This force is obtained by minimizing the homogenous energy equation as

$$
-\left(w_{1} v^{\prime}\right)^{\prime}+\left(w_{2} v^{\prime}\right)^{\prime}+\nabla P(v)=0
$$

The boundary conditions are $v(0), \nabla^{\prime}(), \mathcal{b}()$, and $v^{\prime}(1)$, and $w_{1}$ and $w_{2}$ are constants representing the elasticity and rigidity of the curve, respectively. The potential force is represented as follows [2], [3]:

$$
\int_{0}^{1} P(v(s)) d s
$$

where

$$
P(v(s))=-\nabla I(x, y)^{2}
$$

and $I(x, y)$ is the image intensity.

The potential force used in (3) is the chamfer distance function or the Euclidian distance map. In general the distance potential function can be defined as [2]

$$
P(v(s))=g(d(v(s)))
$$

where $d(v(s))$ is the distance map function.

On the contrary, Cohen and Cohen [2], [3] found that the forces derived from (3) are insufficient for attracting a snake from far distances to the edges. Furthermore, the snake curve will tend to shrink if it is not subjected to counterbalance forces. As a solution, they proposed a second force term as a pressure force that would expand the snake outwards or inwards. The combination of both the 
potential and pressure forces will guide the snake to the desired features and reduce the effect of noise during the movement of the curve. Therefore, the total forces acting on the snake are given as follows [2], [3]:

$$
F=k_{1} \vec{n}(s)-k_{2}(\nabla p /|\nabla p|)(v(s))
$$

where $\vec{n}(s)$ is a unit normal vector to the snake at point $v(s)$ and $k_{1}$ and $k_{2}$ are the constants chosen such that $k_{2}$ is slightly larger than $k_{1}$ for the snake to stop at the strong edges.

Another area of concern is the concavity convergence issue. $\mathrm{Xu}$ and Prince [4]-[6] proposed the gradient vector flow (GVF) field to solve the proper initialization assumptions for the potential fields under concern.

Unlike classical potential fields, the GVF field cannot be thought of as a negative gradient of a potential field because it is formulated directly from the conditions of balance forces. The main idea of GVF is to produce a smooth field at regions with small edge map gradients and to maintain the strength of the gradient of the edge map in the vicinity of the edges. This vector field is defined parametrically as [4], [5]

$$
V(x, y)=\lfloor u(x, y), v(x, y)\rfloor
$$

which minimizes the following energy equation

$$
E=\iint\left(\mu\left(\nabla^{2} u+\nabla^{2} v\right)+|\nabla f|^{2}|V-\nabla f|^{2}\right) d x d y
$$

The second term in (8),i.e., $|\nabla f|^{2}|V-\nabla f|^{2}$, verifies the main concept of GVF: at regions of high edges, the map gradient $\nabla f$ dominates the field: at regions where $\nabla f \rightarrow 0$, the diffusion field $V$ will dominate. To minimize the energy equation, the filed $V$ should satisfy the following homogenous linear Euler partial differential equations [4], [5]:

$$
\begin{aligned}
& \mu \nabla^{2} u-\left(u-f_{x}\right)\left(f_{x}^{2}+f_{y}^{2}\right)=0 \\
& \mu \nabla^{2} v-\left(v-f_{y}\right)\left(f_{x}^{2}+f_{y}^{2}\right)=0
\end{aligned}
$$

A finite difference method was used to solve (9) and (10) iteratively to reach the steady state condition which is the desired diffusion field. The computational complexity of the GVF is considered high compared with the distance field [4]. On MATLAB code using the SGI Indego-2 machine, the computation time for the same image shape is measured as 420 seconds for the GVF algorithm compared with 155 seconds for the distance fields.

GVF provided a smooth varying field in regions far from the edges and maintained the edge gradient in regions near the edges. As a result, a better capture range and a smoother field transition are obtained, particularly in the concave region.

A drawback of the GVF method is reported in the regions of narrow edges in a given image. This method has a tendency to over smooth the field between opposite edges. To improve this performance, generalized GVF (GGVF) was proposed by $\mathrm{Xu}$ and Prince [7]. Both $\mu$ and $\left(f_{x}^{2}+f_{y}^{2}\right)$ which are given in (9) and (10) are replaced by a nonlinear function of $|\nabla f|$.

Although GVS and GGVF are considered very popular methods, one of the negative aspects of these methods is their inability to automatically handle the segmentation and initialization of multiple objects. Chunming et al. [7] addressed this limitation and proposed the edge preserving GVF (EPGVF). The external force in EPGVF is segmented, and a snake curve is initialized in each segment within the capture range of the associated object [7], [8].

Diverse snake algorithms have been proposed for different image processing applications such as segmentation, shape detection, and building extraction [9], [13].

In this paper, a new external force algorithm for driving the snack to the desired borders is proposed. This force is calculated using the isotropic energy diffusion of high intensity pixels on the basis of the extracted edge maps of the image. Isotropic and anisotropic diffusion techniques are widely used in image processing applications. Image smoothing, reconstruction, edge detection, and denoising are common practices in the image processing fields [14], [15]. However, deriving the isotropic diffusion in this paper highlights the contribution of this work towards traditional diffusion methods. The edge map can be computed by different gradient methods [16], [17] or by calculating the image local energy [18], [20].

The results of this paper show that isotropic potential fields are comparable to the GVF and EPGVF in terms of convergence. On the contrary, it has a remarkable improvement over GVF in terms of the capture range. Furthermore, the computational complexity of the proposed method surmounts both the GVF and the EPGVF.

Section II presents the derivation and the optimization of the proposed potential field. Section III shows experimental setup and results. Section IV concludes.

\section{LOCAL ENERGY DIFFUSION FORCE FORMULATION}

\section{A. Energy Diffusion}

The local energy or edge maps extracted from the image contain the point sources, which represent the edges and the corners of the object (Fig. 1). Each point source applied at time $t=0$ and location $\left(x_{i}, y_{i}\right)$ in the image space can be mathematically represented by assuming that it is an energy source:

$$
E_{i}=\left|E_{i}\right| \delta\left(x-x_{i}\right) \delta\left(y-y_{i}\right) \delta(t)
$$

where $E_{i}$ is the energy level (or intensity) of the point source at location $\left(x_{i}, y_{i}\right)$, and

$$
\delta\left(\tau-\tau_{i}\right)=\left\{\begin{array}{rr}
1, & \tau=\tau_{i} \\
0, & \text { elsewhere }
\end{array}\right.
$$

On the basis of such assumptions, each point source will then start diffusing the energy in the image space. The diffusion process of the energy is governed by the following 
2D homogenous diffusion equation (general energy equation):

$$
\nabla^{2} \varphi(x, y, t)-(1 / D)(\partial \varphi(x, y, t) / \partial t)=0
$$

with the initial conditions representing the instantaneous energy at each point source given as

$$
\varphi(x, y, 0)=E_{i}, \quad \text { where } i=1,2, \ldots N
$$

In this proposed method, $D$, which is the diffusion factor, is assumed constant to initiate a linear isotropic diffusion process. Other assumptions may be taken if $D$ is not assumed constant.

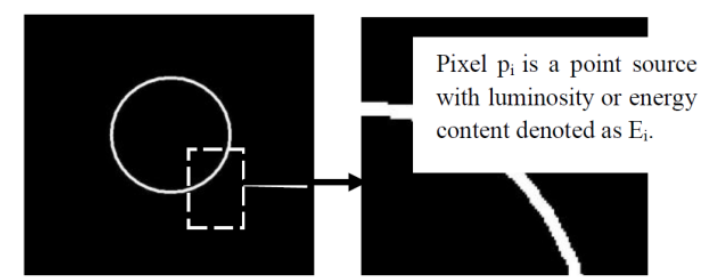

(a)

(b)

Fig. 1. (a) Binary edge map and; (b) zoomed image showing the point source pixels.

Equation (13) is a well-known energy diffusion equation that describes the diffusion of energy in lossless media. The solution to this equation varies according to the boundary and initial conditions provided: such problems are called boundary or initial value problems.

Spatial Fourier transforms can be applied to provide a simple closed form solution by reducing the equation from partial differential to an ordinary differential equation. The Fourier transform of the nth derivative of a $\varphi(x, y, t)$ would be given as

$$
\begin{aligned}
& \frac{\partial^{n}}{\partial x^{n}} \varphi(x, y, t)=\left(2 \pi i k_{x}\right)^{n} \hat{\varphi}\left(k_{x}, k_{y}, t\right) \\
& \frac{\partial^{n}}{\partial y^{n}} \varphi(x, y, t)=\left(2 \pi i k_{y}\right)^{n} \hat{\varphi}\left(k_{x}, k_{y}, t\right)
\end{aligned}
$$

By substituting back in (13), we obtain the following:

$$
\frac{\partial \varphi(x, y, t)}{\partial t}+D(2 \pi)^{2}\left(k_{x}^{2}+k_{y}^{2}\right) \hat{\varphi}(x, y, t)=0
$$

An ordinary differential equation with a solution of the form is then obtained:

$$
\hat{\varphi}(x, y, t)=A \cdot \exp \left(-D(2 \pi)^{2}\left(k_{x}^{2}+k_{y}^{2}\right) t\right)
$$

The inverse Fourier transform of (18) will provide the solution of the initial value diffusion problem defined in (13) and (14) for a point energy source:

$$
\varphi(x, y, t)=\frac{A}{4 \pi D t} \cdot \mathrm{e}^{\frac{-\left(x^{2}+y^{2}\right)}{4 \pi D t}}
$$

By assuming that each point source has an energy value $\left|E_{i}\right|$ and by imposing the condition

$$
\int_{-\infty}^{\infty} \int_{-\infty}^{\infty} \varphi(x, y, t) d x d y=\left|E_{i}\right|
$$

we found that the value of $A$ would be $A=\left|E_{i}\right|$.

For $N$ point sources the total solution is only the superposition given in the following:

$$
\varphi_{\text {tot }}(x, y, t)=\sum_{i=1}^{N}\left(\frac{\left|E_{i}\right|}{k}\right) \mathrm{e}^{\frac{-\left(\left(x-x_{i}\right)^{2}+\left(y-y_{i}\right)^{2}\right)}{k}}
$$

where $\left(x_{i}, y_{i}\right)$, and $\left|E_{i}\right|$ are the position and energy of point source $i$, respectively, and $k=4 \pi D t$.

\section{B. System Optimization}

Before going to the final stage of force derivation, it is wise at this point to mention that the original image is regarded as an initial state of the diffusion process in traditional isotropic and anisotropic diffusion partial differential equation techniques. The filtered version from its temporal evolution is extracted by finite difference schemes. One drawback of all finite difference schemes in image evolutions is the additional blurring of the object borders due to dissipation effects [20], [21]. In this application, the isotropic diffusion is applied over the binary edge maps. Consequently, the dissipative effects from the background over the object borders are eliminated or minimized.

Another blurring source is factor $\mathrm{k}$ in (21), which controls the spread of the diffusion (Fig. 2(a), Fig. 2 (b), and Fig. 2 (c)). The low value of $k$ results in narrow diffusion, and a relatively high value of $k$ would result in severe blur edges.

To reduce the blurring effect and maintain a satisfactory spread of diffusion, the proposed model was optimized as follows:

The constant $k=4 \pi D t$ was optimized experimentally. The best results were found with $P \leq k \leq 15$.

The initial edge map (EM) is super imposed over (21). This approach maintains the sharpness of the object borders and strengthens the field in the near vicinity of edges.

Therefore, the total potential will be

$$
\varphi_{\text {tot }}(x, y, t)=E M+\sum_{i=1}^{N}\left(\frac{\left|E_{i}\right|}{k}\right) \mathrm{e}^{\frac{-\left(\left(x-x_{i}\right)^{2}+\left(y-y_{i}\right)^{2}\right)}{k}}
$$

where $E M$ is the edge map of the image.

The potential in (22) can be further simplified by assuming that each point source has equal energy contents i.e., if

$$
\left|E_{i}\right|=|E| \text { for } i=1, N
$$

Hence, the general form of (22) would be expressed as follows:

$$
\varphi_{\text {tot }}(x, y, t)=E M+\left(\frac{|E|}{k}\right) \sum_{i=1}^{N} e^{\left(\frac{d_{i}(x, y)}{k}\right)}
$$

where $d_{i}(x, y)$ is the distance function between points $(x, y)$ in the image plane and point source $i$ at $\left(x_{i}, y_{i}\right)$.

The potential represented by (24) provides the complete 
solution and the field can be calculated by taking the gradient of $\varphi_{\text {tot }}(x, y, t)$ :

$$
\left\lfloor\varphi_{x}, \varphi_{y}\right\rfloor=\nabla \varphi_{t o t}(x, y, t)
$$

The field vector computed using (25) is used as an external force to drive the snake toward the object borders in the image space. This new proposed scheme is called the local energy diffusion force (LEDF) model.

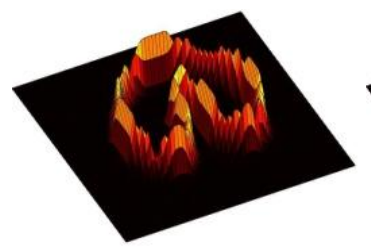

(a)

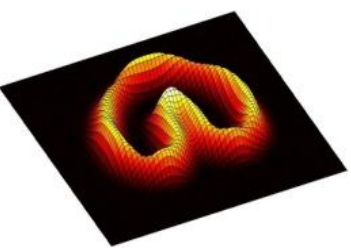

(b)

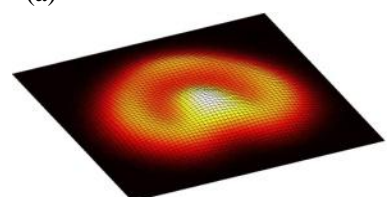

(c)

Fig. 2. Diffusion field effect due to increasing $k$.

\section{EXPERIMENTAL SETUP AND RESULTS}

To compare the proposed LEDF model with the standard GVF and EPGVF snake models, three images are used. Two of the images are edge maps with a resolution of $64 \times 64$ pixels each, and the last image is a grayscale image with a resolution of $350 \times 350$ pixels (Fig. 3 ). $k$ is assumed as $k=12$ for the proposed method and $\mu$ is taken as $\mu=0.2$ for the GVF method. The number of iterations is fixed to 80 iterations for both the GVF and EPGVF models. For all in hand models, the grayscale image is converted to the edge map version by using the well-known Canny edge detection method [16], [17].

The comparison is based on the computation of the required running time to obtain the force field or the speed of calculation between the proposed LEDF model and the GVF and EPGVF models.

All running steps were conducted on a computer using Intel Core ${ }^{\mathrm{TM}} 2$ Duo Processor T5550 (1.83GHz), 3GB RAM, and Windows Vista/SP2 32- bit operating system.

\section{A. Complexity of the Computation of the Field}

The proposed LEDF model is not an iterative method. i.e., the potential is directly computed from an analytical equation that satisfies the energy diffusion criteria. However, the computation would be repeated for every point source at the borders of the object. On the contrary, the calculation would be repeated for each point in the borders domain, and all obtained responses will be algebraically summed. Therefore, the computational complexity, for $\mathrm{N}$ object points would be given as $O(N)$. The computational complexity of the GVF and the gradient vector flow (VFC) are given in the vicinity of $O\left(N^{2}\right)$ [22].

The time elapsed for the force converging process, for every model, is recorded and then averaged for 10 runs. The shapes given in Fig. 3 are used in this process, and Table I shows the calculated average time. The obtained results demonstrate the computational outperformance of the proposed model over both the GVF and EPGVF models.
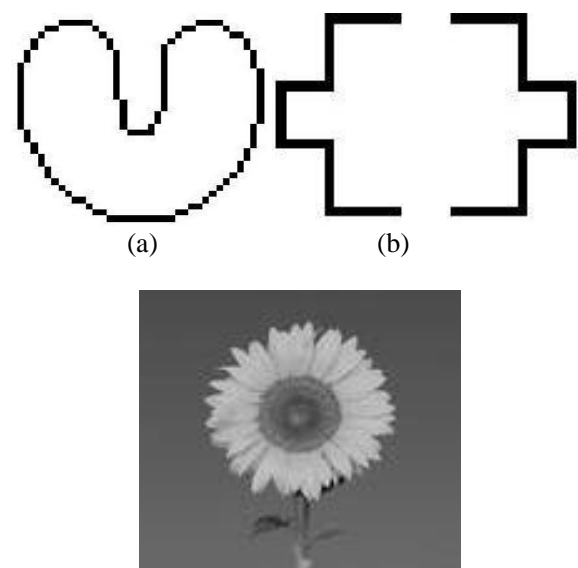

(c)

Fig. 3. (a) The concave shape, (b) the room shape, and (c) the gray scale image.

TABLE I: TIME RECORDED TO CALCULATE THE FORCES FOR LEDF, GVF, AND EPGVF

\begin{tabular}{|l|l|l|l|}
\hline model & Fig. 3 (a)/ second & Fig.3 (b)/ second & Fig. 3 (c)/second \\
\hline LEDF & 0.004 & 0.0031 & 0.045 \\
\hline GVF & 0.21 & 0.23 & 2.6 \\
\hline EPGVF & 0.29 & 0.27 & 3.4 \\
\hline
\end{tabular}

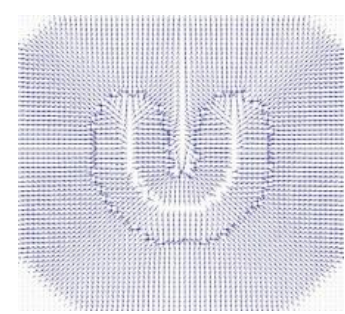

(a)

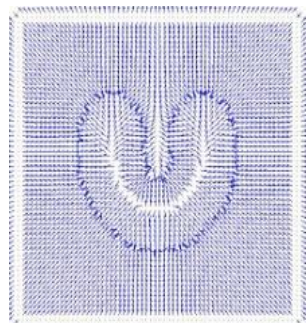

(c)

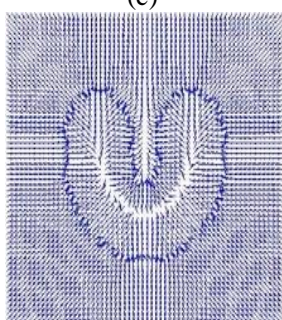

(e)

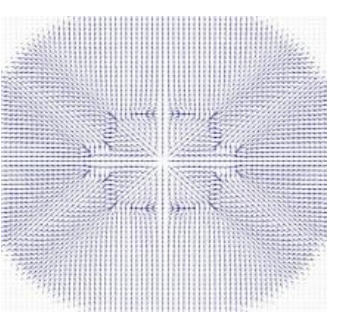

(b)

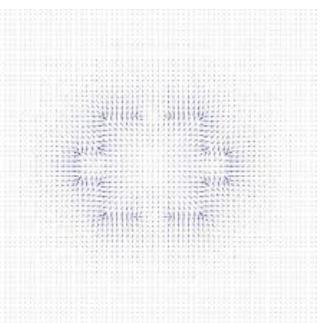

(d)

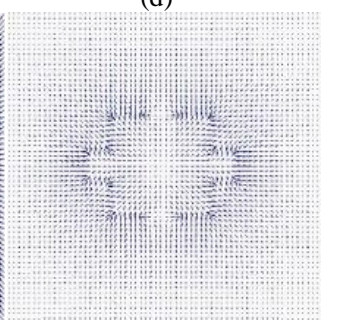

(f)
Fig. 4. The field calculated for the shapes in Fig. 3. (a), (b): the LEDF field; (c), (d): the GVF field; (e), (f): the EPGVF field.

\section{B. Capture Range and Convergence to Concavities}

The second comparison stage would include the capture range and the convergence to concavities. Fig. 4 shows the field obtained using the proposed LEDF method compared with the GVF and EPGVF methods. The images shown in Fig. 3(a); and Fig. 3(b) are also used here for comparison 
purposes. As shown in Fig. 4, the proposed LEDF method produced a high capture range field extending far from the object borders compared with the field obtained by the GVF or EPGVF.

This wide capture range would help solve the problems associated with curve initialization and convergence. This result would clarify the more effective processing technique performed in the LEDF model.

To compare the convergence to concavities parameter, Fig. 5(a) and Fig. 5(b) show the snake initialization and its convergence progress to the boundaries utilizing the LEDF method.

On the contrary, Fig. 6(a), Fig. 6(b), and Fig. 6(c) show the comparison results of the different initialization locations for all the mentioned methods.

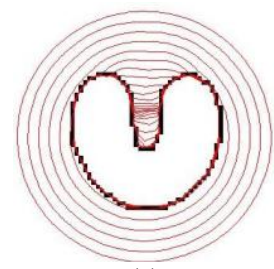

(a)

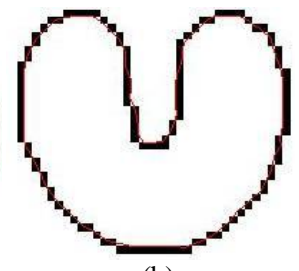

(b)
Fig. 5. (a) Snake initialization and convergence of the LEDF snake for the shape in Fig. 3(a).
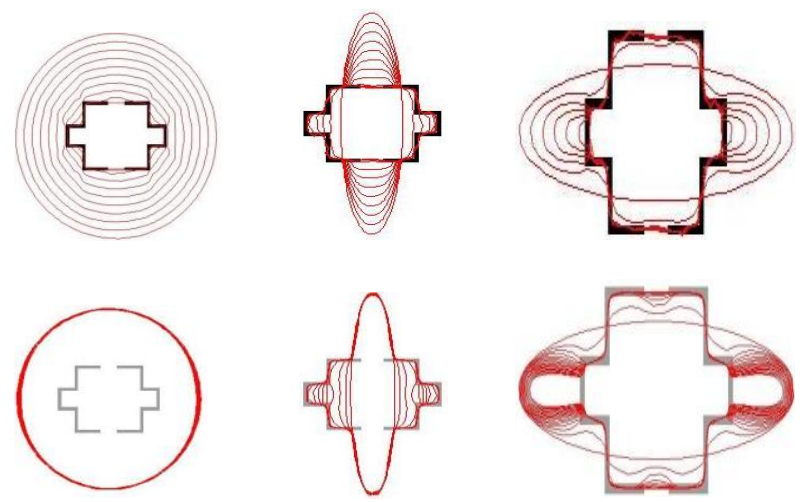

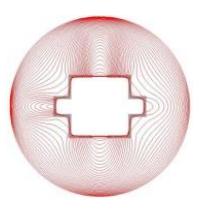

(a)

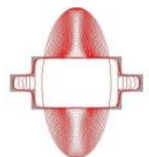

(b)

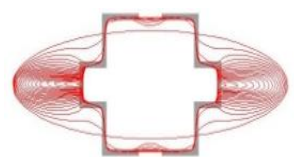

(c)
Fig. 6. (above) The convergence of the LEDF snake for the object in Fig 3(b) for different initializations, (middle) the convergence of the GVF snake, and (bottom) the convergence of the EPGVF.
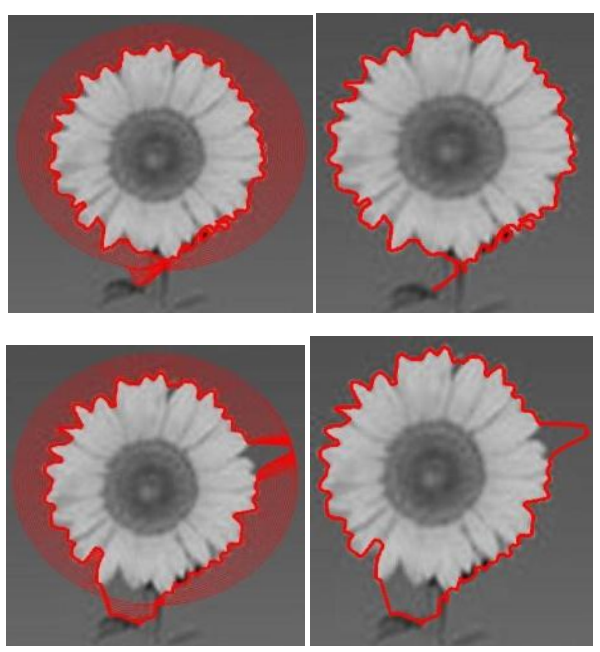

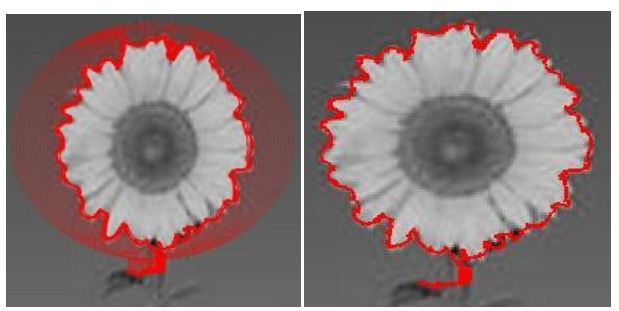

(a)

(b)

Fig. 7. Above: (a) the LEDF snake progress and (b) the final snake curve. Middle: (a) the GVF snake progress and (b) the final snake curve. Bottom: (a) the EPGVF snake progress and (b) the final snake curve. (in all methods, the Canny edge detector is used to derive the edge maps).

As inferred from Fig. 6, GVF has failed to converge to the object borders in Fig. 6(a), Fig. 6(b) and Fig. 6(c).

This finding may be attributed to the weak field obtained by the GVF method at areas that are far from the object borders. The outperformance of the LEDF method would support the claim of the effectiveness of the model.

Finally, the real grayscale image shown in Fig. 3(c) was also used to compare the performance of the LEDF in contrast to both the GVF and the EPGVF. The results given in Fig. 7 (a) and Fig. 7(b) clarify the precise convergence of the LEDF over the GVF and EPGVF methods.

\section{FUTURE WORK}

In this paper, the external force field is deployed by a simple isotropic closed form solution. This solution provided satisfactory snake convergence with great improvements in computational complexity. However, this isotropic solution may cause border migration at high curvature regions. Therefore, the anisotropic solution to the diffusion equation achieved via the separation of variables may be proposed to mitigate the borders migration. The applicability of the anisotropic solution and their computational complexity are questions that will be answered in future works.

\section{CONCLUSION}

In this paper a new external force is proposed for snake convergence. The new proposed force is computed from the energy diffusion potential. This potential can be calculated by considering the edge points as energy sources (point sources) and by calculating the diffusion of this energy in the image domain. The diffusion equation of the proposed model is calculated from a general distance function. The computational complexity of the proposed LEDF snake increases linearly with the number of point sources available in the image; however, the proposed method outperforms both the GVF and EPGVF methods. The proposed LEDF snake provided a good capture range and was able to converge faster into the desired object concavities. Future studies will be performed to improve the convergence, and the performance of the LEDF snake on more complex realistic images.

\section{REFERENCES}

[1] M. Kass, A. Witkin, and D. Terzopoulos, "Snakes: Active contour models," International Journal of Computer Vision, vol. 1, pp. 321333, 1988. 
[2] L. D. Cohen and I. Cohen, "Finite element methods for active contour models and balloons for 2D and 3D images," IEEE Transactions on Pattern Analysis and Machine Intelligence, pp. 1131-1147, Nov. 1993.

[3] L. D. Cohen and I. Cohen, "A finite element method applied to new active contour models and $3 \mathrm{D}$ reconstruction from cross sections," Proceeding, third International Conference on Computer Vision, pp. 587- 591, 4-7 Dec 1990.

[4] C. Xu and J. L. Prince "Snakes, shapes, and gradient vector flow," IEEE Transaction on Image Processing, pp. 359-369, Mar. 1998.

[5] C. Xu and J. L. Prince, "Gradient vector flow: A new external force for snakes," IEEE Computer Society Conference on Computer Vision and Pattern Recognition, pp. 66-71, Jun. 17-19, 1997.

[6] C. Xu and J. L. Prince, "Generalized gradient vector flow external forces for active contours," Elsevier, Science Direct, Signal Processing, vol. 71, pp. 131-139, 1998.

[7] L. Chunming, L. Jundong, and D. F. Martin, "Segmentation of edge preserving gradient vector flow: An approach toward automatically initializing and splitting of snakes," in Proc. the 2005 IEEE Computer Society Conference on Computer Vision and Pattern Recognition (CVPR'05), 2005, pp. 1063-1069.

[8] A. Basavaraj and P. K. Kulkrani, "External force for deformable models in medical image segmentation: A survey," Signal and Image Processing: An International Journal (SIPIJ), vol. 2, no. 2, pp. 82101, June 2011.

[9] J. Tang, S. Millington, S. T. Action, J. Crandall, and S. Hurwitz, "Ankle cartilage surface segmentation using directional gradient vector flow snake," in Proc. Int. Conference Image Processing, 2004, pp. 2745-2748.

[10] J. R. Cheng and S. W. Foo, "Dynamic directional gradient vector flow for snakes," IEEE Transaction on Image Processing, vol. 15, no. 6, pp. 1563-1571, 2006.

[11] M. Kabolizade, H. Ebadi, and S. Ahmadi, "An improved snake model for automatic extraction of buildings from urban aerial images and LiDAR data," Computers, Environment and Urban Systems, vol. 34, pp. 435-441, 2010.

[12] J. Li and J. H. Xu, "Contour segmentation algorithm of multi-scale GVF snake," in Proc. the 2006 IEEE International Conference on Mechatronics and Automation, Luoyang, China, 2006, pp. 537-542.

[13] M.-A. Cahrmi, S. Derrode, and F. Ghorbel, "Fourier-based geometric shape prior for snakes," Pattern Recognition Letters, vol. 29, pp. 897 904, 2008.

[14] J. Weickert, Anisotropic Diffusion in Image Processing, Stuttgart: Teubner, Jan. 1998, vol. 1, pp. 43-45.
[15] P. Perona and J. Malik, "Scale-space and edge detection using anisotropic diffusion," IEEE Transactions on Pattern Analysis and Machines Intelligence, vol. 12, no. 7, pp. 629-639, 1990.

[16] E. N. Nejad, "Edge detection techniques: Evaluations and comparisons," Applied Mathematical Sciences, vol. 2, no. 31, pp. 1507-1520, 2008.

[17] N. Senthilkumaran and R. Rajesh, "Edge detection techniques for image segmentation - a survey of soft computing approaches," International Journal of Recent Trends in Engineering, vol. 1, no. 2, 2009, pp. 250-254

[18] P. D. Kovesi, "Invariant measures of image features from phase information," PhD thesis, Dep. Psychology, The University of Western Australia, May 1996.

[19] B. J. Robbins, "The detection of 2D image features using local energy," $\mathrm{PhD}$ thesis, Dep. Computer Science, The University of Western Australia, Aug. 1996.

[20] P. D. Kovesi, "Phase congruency detects corners and edges," Proc. VIIth Digital Image Computing: Techniques and Applications, Sydney, pp. 309-318, 2003.

[21] P. D. Kovesi, "Edges are not just steps," in Proc. 5th Asian Conference on Computer Vision, ACCV2002, Melbourne, Australia, pp. 1-6.

[22] B. Li and S.T. Acton, "Active contour external force using vector field convolution for image segmentation," IEEE Transactions on Image Processing, vol. 16, no. 8, pp. 2096-2106, Aug. 2007.

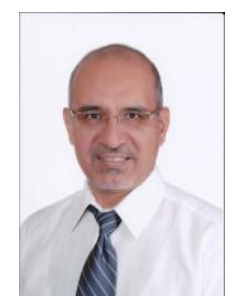

Mahdi Nisirat is currently an assistant professor at the Faculty of Engineering Technology, Department of Communications Technology, Albalqa Applied University. He holds a PhD degree from Universiti Kebangsaan Malaysia, Malaysia; an MSc degree in electrical engineering from the University of Texas at Arlington, USA; and a BSc degree in mathematics physics (double major) from King Abdul Aziz University, Saudi Arabia. His current research interests include cellular path loss prediction and optimization, microwave propagation modeling, MIMO systems and techniques, and image processing techniques and optimizations.

$\mathrm{He}$ is currently the IEEE COMSOC head of the Department of Communications Technology. 